\title{
A typical Presentation of Lung Abscess
}

\section{UA Sheikh MRCP(UK), R Darracott, A Taylor FRCR}

\section{BACKGROUND}

A lung abscess is a localised suppurative infection in the lung parenchyma, characterised by the formation of a necrotic cavity $>2 \mathrm{~cm}$ enclosed by a fibrous wall. Classic symptoms are onset of high-grade pyrexia (>38.5), productive cough with putrid sputum and presence of risk factors. ${ }^{1}$

Lung abscesses are associated with aspiration of gastric or oropharyngeal contents, which arise most often in patients with altered consciousness or dysphagia. Abscess formation has also been shown to occur distally to obstructing tumours or foreign bodies, as well as secondary to septic emboli or infected pulmonary infarcts. Immunocompromised patients are also at risk. ${ }^{2,3}$

In abscesses found in adults over the age of 50 years there was a preceding pneumonia in up to $40 \%$ of cases, and almost $50 \%$ were associated with lung tumours. ${ }^{4,5}$

\section{CASE PRESENTATION}

A 78-year-old gentleman presented to his general practitioner with a dry cough that had been ongoing for two months, without shortness of breath or chest pain. On further questioning he admitted to a long history of smoking. Examination revealed crepitations in his left lower base, but was otherwise unremarkable. The patient's past medical history consisted of pre-diabetes mellitus, benign prostate hypertrophy, iron-deficiency anaemia, asthma and colon carcinoma which was resected 13 years ago.

Given his history of smoking, the patient met the 'direct access' criteria for chest $\mathrm{x}$-ray imaging and this was performed within two weeks (See Fig. 1). ${ }^{7}$ The x-ray was reported by a radiology consultant who noted "a new spiculated opacity in the right lower zone medially...a

\begin{tabular}{|l|}
\hline Differential diagnosis of Lung Mass \\
Hyperdense Mass \\
- Primary lung cancer \\
- Secondary metastases \\
- Granuloma \\
- Bone-Forming: osteosarcoma, chondrosarcoma \\
- Dystrophic calcification: papillary thyroid \\
carcinoma, giant cell tumour of bone, synovial \\
sarcoma \\
Cavitating Mass \\
- Cavitating malignancy (primary or secondary) \\
- Vascular: Pulmonary infarct \\
- Trauma: Pneumatocele \\
O Tubection \\
o Cavitating pneumonia \\
O Septic pulmonary emboli \\
\hline
\end{tabular}

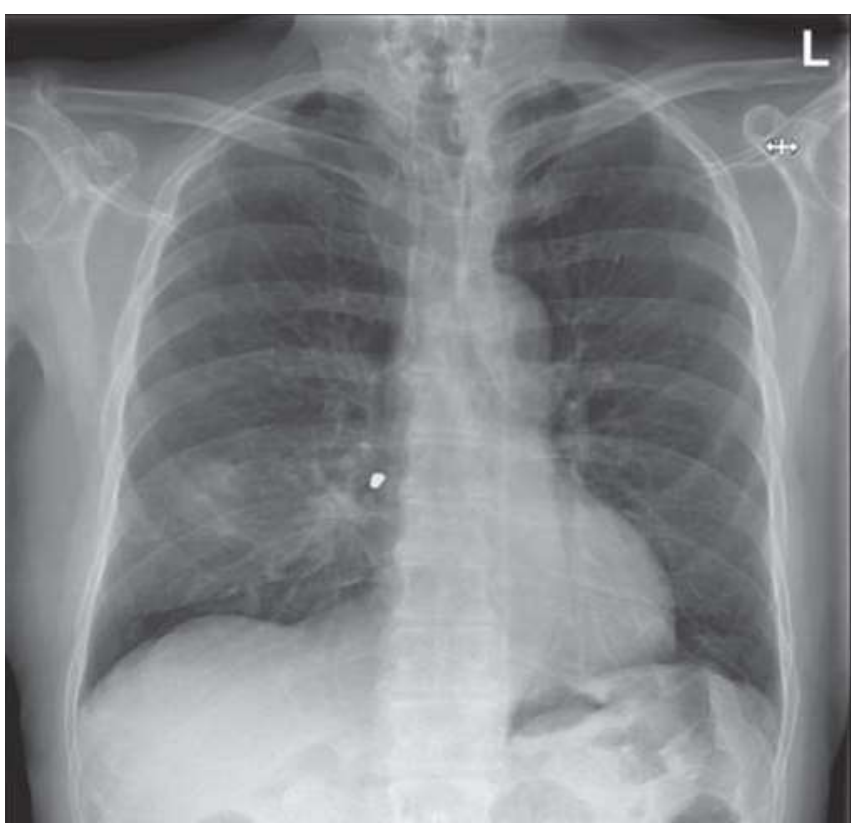

Figure 1: The initial chest $\mathrm{x}$-ray ordered by the patient's general practitioner.

further lung opacity is seen in the right lower zone more laterally, measuring $36 \mathrm{~mm}$. There is a new calcified nodule in the right hilar region."

Further investigation with a CT thorax, abdomen and pelvis (CT-TAP) was recommended and organised for within two weeks, as per the suspected cancer pathway. ${ }^{7}$

\section{INVESTIGATIONS}

The patient's blood results were as follows:

\begin{tabular}{|l|l|}
\hline $\mathrm{Hb}$ & $117(\downarrow)$ \\
\hline MCV & 87.0 \\
\hline WCC & $11.9(\uparrow)$ \\
\hline Neutrophils & $9.5(\uparrow)$ \\
\hline
\end{tabular}

Liver, bone and renal profiles were unremarkable.

\section{TREATMENT}

The gentleman's CT-TAP was performed, revealing an $8 \mathrm{~mm}$ metal foreign body at the right hilum and a $25 \mathrm{~mm}$ fluid-filled lesion in the right middle lobe, which was collapsed (See Fig. 2). The scan was discussed in the lung multidisciplinary meeting and urgent endoscopy was arranged. At this point the patient was informed of the CT scan results and mentioned he had noticed a missing dental filling several months ago. He was commenced on antibiotics as per the trust's microbiology protocol.

Bronchoscopy confirmed the presence of dental amalgam at the entrance to the right middle lobe, however attempt to remove under suction was unsuccessful and unfortunately pushed the foreign object into the left lower lobe where it was unable to be retrieved. 


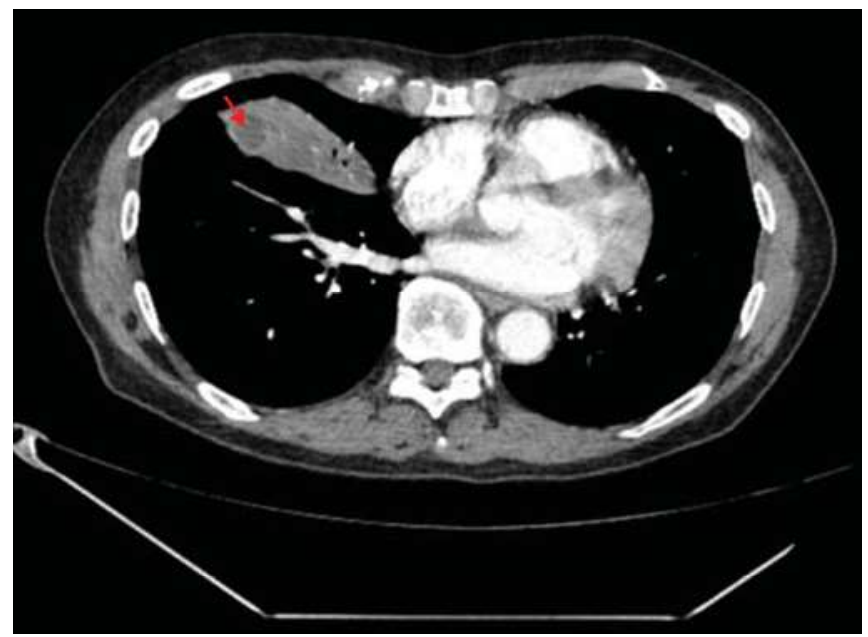

Figure 2: This slice demonstrates the radiological findings best; the right middle lobe collapse is clearly visible with the $25-\mathrm{mm}$ fluid-filled lesion indicated by a red arrow.

\section{OUTCOME AND FOLLOW-UP}

The patient was referred to a neighbouring hospital the following day for rigid bronchoscopy where the dental amalgam was successfully removed and he was discharged later the same day. He will continue on antibiotics for 6-8 weeks with regular chest $\mathrm{x}$-rays to monitor treatment response.

\section{LEARNING POINTS}

- The radiologic appearances of abscess are cavitation with air-fluid level on a background of segmental or lobular consolidation. ${ }^{6}$
- Chronic cough often proves difficult to evaluate and hence manage. The National Institute of Clinical Excellence (NICE) recommends obtaining an $\mathrm{x}$-ray on all patients aged 40 and over if they have a smoking history and cough.?

- In the treatment of lung abscesses cavity closure takes an average of four weeks. Serial chest $\mathrm{x}$-rays are utilised to monitor therapeutic response. ${ }^{2}$

\section{REFERENCES}

1. Bartlett JG. Anaerobic bacterial infections of the lung. Chest 1987 Jun;91(6):901-9.

2. Kasper D, Fauci A, Hauser S, Longo D, Jameson J, Loscalzo J. Harrison's principles of internal medicine. 19th ed. Maidenhead: McGraw-Hill Education, 2015:813-815.

3. Thomson EC, Lynn WA. Septic thrombophlebitis with multiple pulmonary abscesses. Lancet Infect Dis 2003;3:86.

4. Pohlson EC, McNamara JJ, Char C, et al. Lung abscess: a changing pattern of disease. Am J Surg 1985;150:97-101.

5. Hirshberg B, Sklair-Levi M, Nir-Paz R, et al. Factors predicting mortality of patients with lung abscess. Chest 1999; 115:746-750.

6. Groskin SA, Panicek DM, Ewing DK, et al. Bacterial lung abscess: a review of the radiographic and clinical features of 50 cases. J Thorac Imaging 1991 Jul;6(3):62-7.

7. NICE (2015). Suspected cancer: recognition and referral [NG12]. Available at: https://www.nice.org.uk/guidance/ ng12/chapter/1-recommendations-organised-by-site-ofcancer\#lung-and-pleural-cancers (accessed 30.06.19).

Correspondence to: uasheikh1@gmail.com

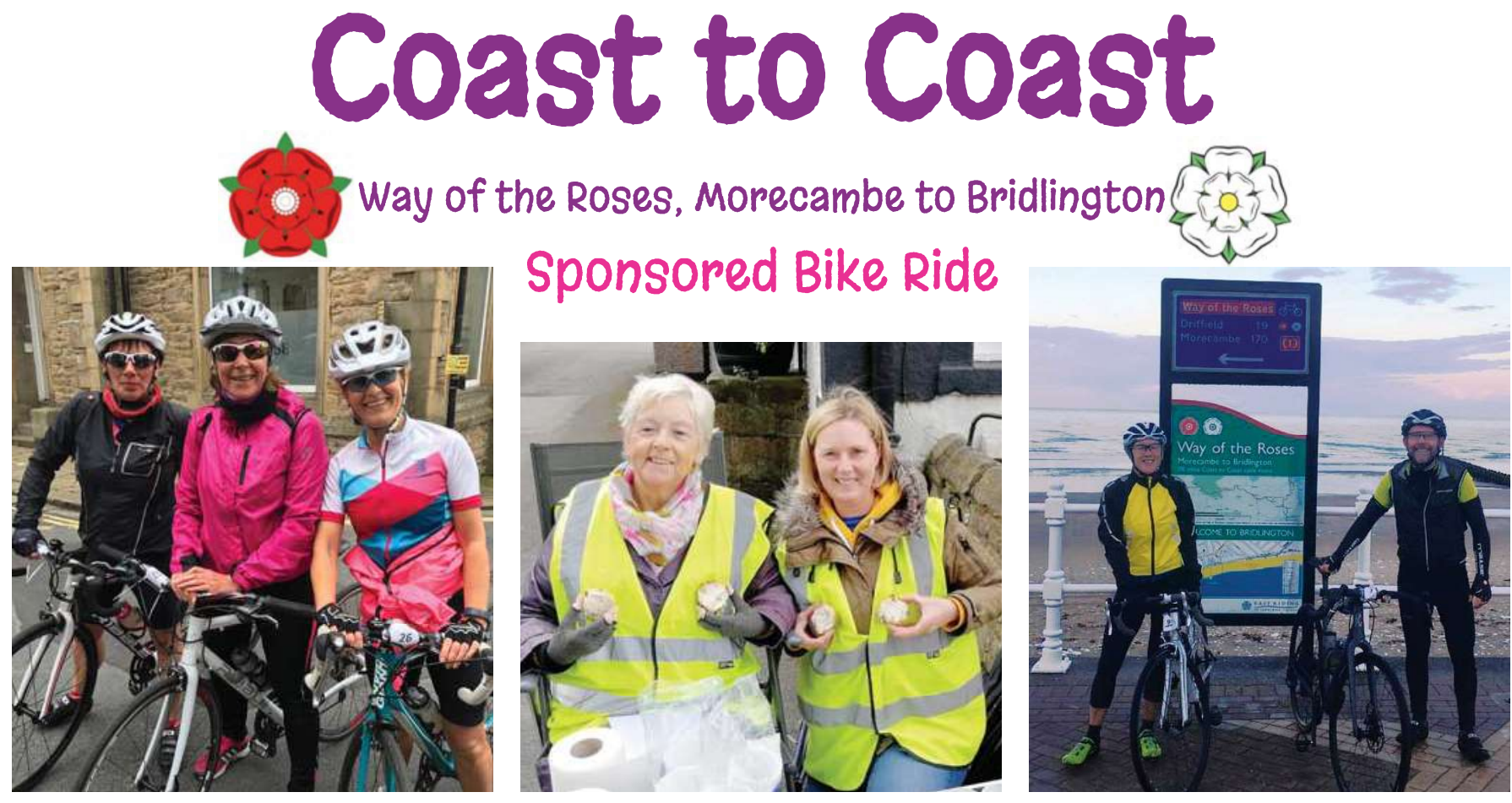

So far, it is looking like we have raised almost $£ 15,000$ towards our target of $£ 40,000$ towards the Head and Neck Cone Beam CT scanner. 